\title{
The Effect of SC vehicles on the Characteristics of Single-lane Traffic Flow
}

\author{
Qingguo Zhou \\ School of North China Electric Power University Baoding, Baoding 071000, China \\ 1768316219@qq.com
}

Keywords: Nagel-Schrekenberg; Self-driving with Cooperating; Computer Simulation

\begin{abstract}
On the basis of the Nagel-Schrekenberg(NS)model, we first study the effect of SC (Self-driving with cooperating)system on the characteristics of single-lane flux under periodic boundary conditions. There are two models of vehicles on the lane(NS and SC). The influence of the proportion of SC vehicles and the Specified headway on the flux is studied by using computer simulation.
\end{abstract}

\section{Introduction}

In this model ,we select the single-lane Highway SR-520 as the object of study which is composed by the one-dimensional discrete lattice chain whose length is 7527 cell,that is, each grid point is equivalent to 3yards.The total length of the road is 12.83miles.At each moment, the lattice point may be occupied by the vehicle, the vehicle occupies two lattice points, then the corresponding actual length is about 6yards, and the maximum velocity is $v_{\max }=9$ cells $/ \mathrm{s}$ (road velocity limit $60 \mathrm{mph}$ ), the corresponding actual velocity is $55 \mathrm{mph}$.Each type of vehicle state is represented by its own velocity $v \quad 0 \leq v \leq v_{\max }$. With $x_{i}(t)$ represents the ${ }^{i}$ vehicle at the $t$ time of the rear position, $d_{i}(t)=x_{i+1}(t)-x_{i}(t)-l_{i}$ represents the distance between the vehicle $(i+1)$ and the immediately preceding neighboring vehicle $(i+1), T=d_{i} / v_{i}$ indicates the vehicle time In each evolutionary step $t \rightarrow t+1$, some vehicles perform the velocity and position according to the evolution rule of the NS model and others according to the evolution rule of the SC model ${ }^{[1]}$.

\section{The Updating Rules of SC State Evolution ${ }^{[2,3]}$.}

(1) Acceleration or deceleration process: $v_{i}(t+1) \rightarrow \min \left(v_{\max },\left[d_{i} / T\right]\right)$

(2) Random selection process with probability $p$ :

$v_{i}(t+1) \rightarrow \max \left(v_{i}(t)-1,0\right)\left(\right.$ ifd $_{i} / T<v_{\max }, p=\left[d_{i} / T\right]-d_{i} / T ;$ ifd $\left._{i} / T>v_{\max }, p=0\right)$

(3) Location Update: $x_{i}(t+1) \rightarrow x_{i}(t)+v_{i}(t+1)$

Where $\left[d_{i} / T\right]$ represents the smallest integer but not less than $d_{i} / T$.

\section{Computer Simulation and Numerical Analysis}

In the simulation ${ }^{[4]}$, the length of the lane is $L$, and if the total number of vehicles on the lane is $N$, we will get that the total density of the vehicle $\lambda=N / L$; the total lane occupancy (the ratio of the number of grid points occupied by all vehicles to the lane length) $c=2 N / L$. In order to consider the mixing degree of SC vehicles, SC vehicle mixing ratio coefficient $R$ is introduced, $0.0 \leq R \leq 1.0$. When $R=0.0$, there is no SC vehicle in the lane, while $R=1.0$, there are only SC vehicles in the lane.The average velocity at time $t$ is defined as $\bar{V}_{t}=\frac{1}{N} \sum_{i=1}^{N} v_{i}(t)$; the average flux is $J_{t}=\bar{V}_{t} \times \lambda$.

The simulation adopts the periodic boundary condition. At the initial time, the vehicle is randomly mixed in the lane according to the mixed proportion coefficient $R$, and the initial velocity is 0 ,and the 
time steps of each model evolution is 80000 steps, the velocity of each step in the last 3000 steps is recorded, then the 3000 velocity values are averaged to obtain the velocity $V=1 / T \sum_{t=t_{0}}^{t_{0}+T-1} \bar{V}_{t}$, average flow rate $J=V \times \lambda$,.In order to eliminate the influence of random initial position ,then we take the average of 30 samples $^{[5]}$.

The Effect of the $R$ of SC Vehicle on Traffic Flow.

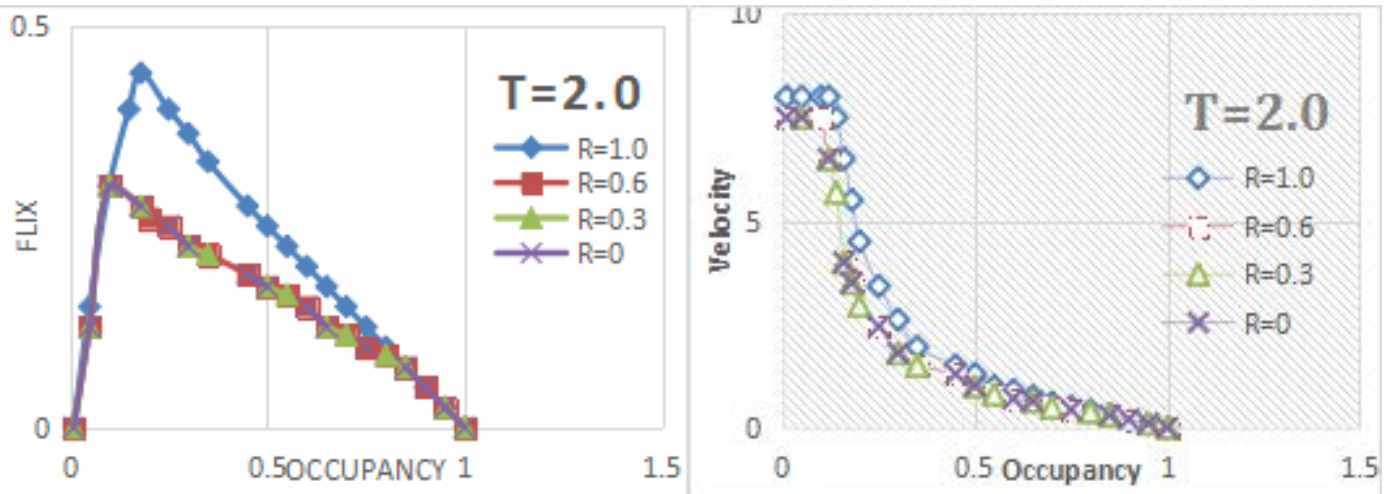

Figure.1 the relationship between the flow and occupancy ratio ,the velocity and occupancy ratio when the ratio $R=0.0,0.3,0.6,1.0,1.0$ for $T=2.0$

When $R=0.0$, there is no SC vehicle in the lane, which is equivalent to the flux under the NS model,It can be seen from Fig. 1 that when $R \neq 1.0$, the flow rate and velocity are not affected by $R$, the critical occupancy $c=0.1$, the maximum flow rate is $J \approx 0.317$, the maximum average velocity $\bar{V} \approx 7.5 \mathrm{cell} / \mathrm{s}$, and when $R=1$, the critical occupancy rate, the flow rate and the velocity of the lane are both greatly improved.The critical occupancy increased to 0.13 , the maximum flow increased to 0.314 , and the maximum average velocity became $\bar{V} \approx 8 \mathrm{cell} / \mathrm{s}$

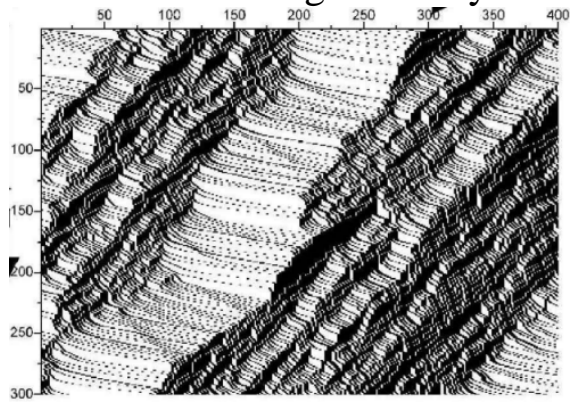

(a) $\mathrm{R}=0.0$

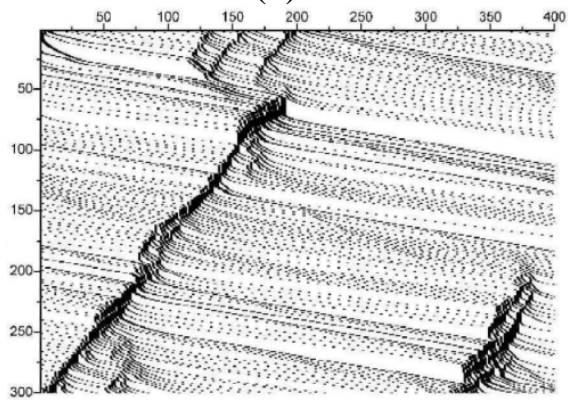

(c) $\mathrm{R}=0.6$

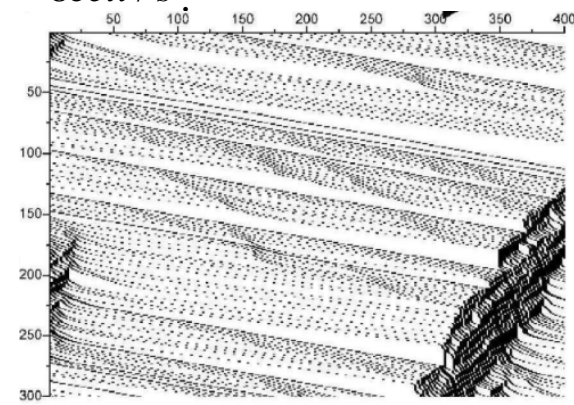

(b) $\mathrm{R}=0.3$

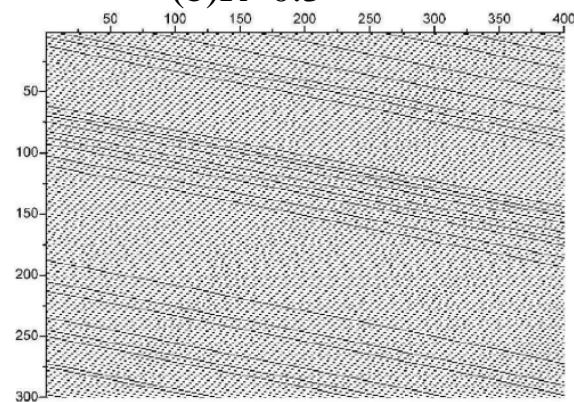

(d) $\mathrm{R}=1.0$

Figure.2. The spatiotemporal evolution pattern of SC vehicle and NS vehicle mixed with $T=2.0, c=0.3$ and $R=0.0,0.3,0.6,1.0$

Although when $R \neq 1.0$, the flow and velocity are not affected by $R$, but with the increase of $R$, the partial blockage can be dissipated gradually.Fig.2 shows the spatiotemporal evolution pattern of SC vehicle and NS vehicle mixed with $T=2.0, c=0.3$ and $R=0.0,0.3,0.6,1.0$.

In the Fig.2, the evolution of 400 lattice points from 79,700 to 80,000 times is presented. The horizontal axis represents the vehicle position, the vertical axis represents time, and the vehicle 
moves from left to right. The black dot indicates the vehicle position, and the white point indicates the space point. The black area indicates a partial blockage of the vehicle and the gray area indicates the free movement of the vehicle.Fig.2(a) shows the spatiotemporal pattern when $R=0.0$, that is, no SC vehicles on the lane, NS vehicles only which shows that local congestion is more serious. Fig.2 (b) and (c) correspond to the case of $R=0.3$ and $R=0.6$. As $R$ increases, the clogging gradually dissipates. When $\mathrm{R}=1$, there are all SC vehicles on the road, when the blocking completely dissipated, the vehicle is in smooth driving state, as shown in Fig.2 (d).

\section{The Effect of the T of SC Vehicles on Traffic Flow.}
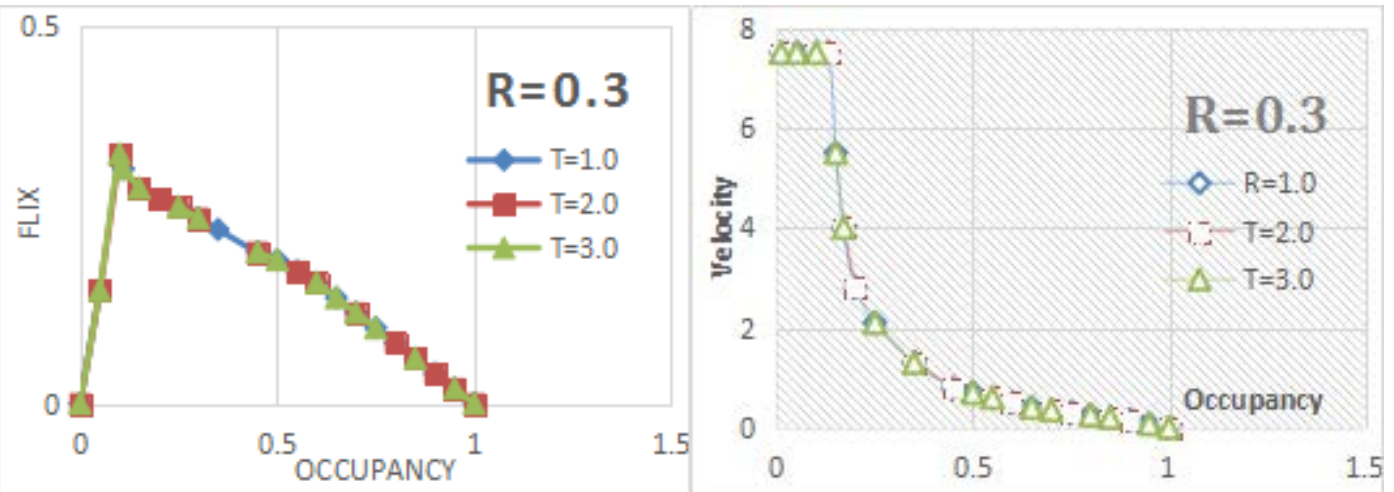

Figure.3. the relationship between the flow and occupancy ratio ,the average speed and occupancy ratio when the ratio $T=1.0,2.0,3.0$ for $R=0.3$

Considering the influence of on flux characteristics of SC vehicles ${ }^{[6]}$. With increases from 1.0 to 3.0,Fig. 3 shows the relationship between traffic and occupancy,velocity and occupancy. The main parameters are as follows:.It can be seen from Fig.3 that has no effect on the flux and average velocity of the mixed flux under the determined $R$.

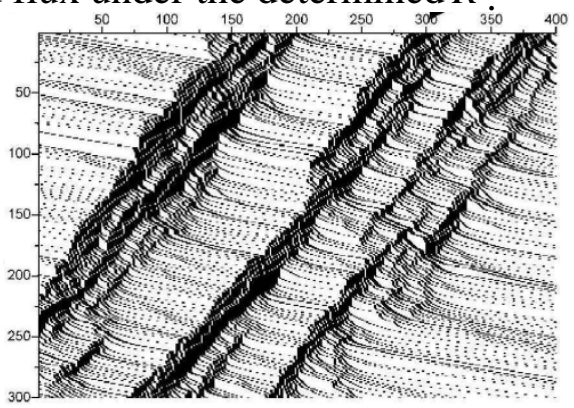

(a) $\mathrm{T}=1.5$

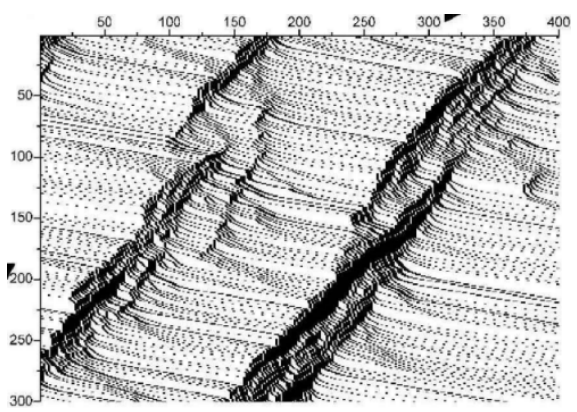

(b) $\mathrm{T}=2.0$

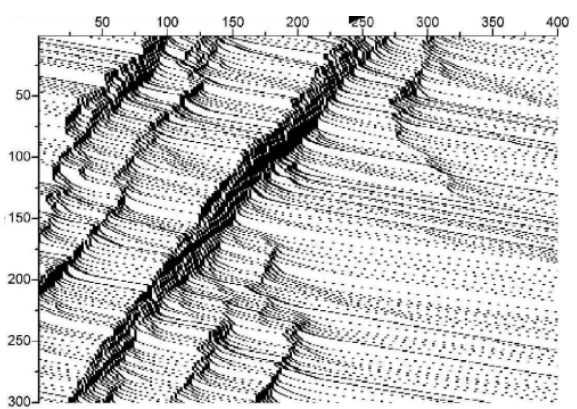

(c) $\mathrm{T}=3.0$

Figure.4: The spatiotemporal evolution pattern at $R=0.5, \quad c=0.3, T=1.5,2.0,3.0$

In the Fig.4, the evolution of 400 lattice points from 79,700 to 80,000 time steps is given. It can be seen from the comparison of (a), (b) and (c) that there is local blockage when $T$ is equal to 1.5, 2.0, 3.0, but as $T$ increases, the clogging zone gradually decreases ${ }^{[7]}$. 


\section{Summary}

In this chapter, based on the NS model of cellular automaton, a flux model of vehicle with SC system is established in order to study the influence of SC vehicle on flux The computer simulation results are given: if there are all SC in the single lane, the critical occupancy rate decreases with the increase of $T$; when the occupancy rate is less than the critical flow rate,average velocity and vehicle distance independent; in contrast,the flow rate and the velocity decrease with the increase of $T$. when the occupancy rate is less than the critical occupancy rate, Flow and velocity has nothing to do with the $T$; in contrast,the flow rate and the velocity decrease with the increase of $T$. When the SC vehicle is mixed with the NS vehicle, the critical occupancy rate has nothing to do with the $R$ and $T$ of the SC vehicle .In the free motion phase, the mean velocity is not affected by $R$. In the congestion phase, the flow is almost unaffected by the $R$. The average velocity increases with the increase of the $R$. The congestion of the flux in the lane will gradually dissipate with the increase of the $R$ and $T$ of the SC vehicle.

\section{References}

[1] CHEN Caixia; The Modeling and Simulation Research on Traffic Capacity of Highways under the Effect of Speed Dispersion (June ,2014)

[2] TANG Liantao ; Modeling and Simulation of the Traffic Flow in Regional Road Network based on Cellular Automaton (May 2012)

[3] WU Dayan ,TAN Huili , KONG Linjiang , LIU Muren ;Study on a three-lane cellular automata traffic flow model ,JOURNAL OF SYSTEMS ENGINEERING ,Vol.20 No.4 Aug.2005

[4] WU Kefei;The Studies of Cellular Automata Models for Mixed Traffic Flow (May ,2007)

[5] HU Jiajun; Scheduling of Connected Autonomous Vehicle on Highway Lanes(January,2013)

[6] QIU Xiaoping,MA Lina,ZHOU Xiaoxia,YANG Da ;The Mixed Traffic Flow of Manual-automated Driving Based on Safety Distance,Journal of Transportation Systems Engineering and Information Technology,Vol.16 No.4 ,August 2016

[7] YANG Liuqing; Research on Traffic Flow Characteristics of Freeway based on Cellular Automata(December, 2014) 